\title{
Remote-controlled optics experiment for supporting senior high school and undergraduate teaching
}

S. H. Choy, K. L. Jim, C. L. Mak, C. W. Leung

S. H. Choy, K. L. Jim, C. L. Mak, C. W. Leung, "Remote-controlled optics experiment for supporting senior high school and undergraduate teaching," Proc. SPIE 10452, 14th Conference on Education and Training in Optics and Photonics: ETOP 2017, 1045241 (16 August 2017); doi: 10.1117/12.2266253

SPIE Event: 14th Conference on Education and Training in Optics and Photonics, ETOP 2017, 2017, Hangzhou, China 


\title{
Remote-controlled optics experiment for supporting senior high school and undergraduate teaching
}

\author{
S.H. Choy, K.L. Jim, C. L. Mak, C. W. Leung \\ Department of Applied Physics, The Hong Kong Polytechnic University, Hung Hom, Hong \\ Kong, People's Republic of China
}

\begin{abstract}
This paper reports the development of a remote laboratory (RemoteLab) platform for practising technologyenhanced learning of optics. The development of RemoteLab enhances students' understanding of experimental methodologies and outcomes, and enable students to conduct experiments everywhere at all times. While the initial goal of the system was for physics major undergradutes, the sytem was also made available for senior secondary school students. To gauge the impact of the RemoteLab, we evaluated two groups of students, which included 109 physics $1^{\text {st }}$-year undergraduates and 11 students from a local secondary school. After the experiments, evaluation including questionnaire survey and interviews were conducted to collect data on students' perceptions on RemoteLab and implementation issues related to the platform. The surveys focused on four main topics, including user interface, experiment setup, booking system and learning process. The survey results indicated that most of the participants' views towards RemoteLab was positive.
\end{abstract}

Keywords: Remote experiment, experimental experiences, undergraduates, K12 education, development.

\section{INTRODUCTION}

Laboratory teaching has always been a major component in science education [1]. The processes of making observations, performing systematic and quantitative investigations, data collection and analysis, are the skills fundamental to the training of all science subjects. Performing experiments also serve to reinforce students' classroom learning experiences. Well-controlled laboratory settings can provide students with first-hand experiences to the relevant scientific phenomena and verify their knowledge acquired from textbooks.

In general, experiment teachings are carried out during traditional laboratory sessions. Students are asked to perform well-controlled experiments in pre-defined time slot. This arrangement allows a large number of students to perform the experiments together with the supervision of only a few instructors. However, traditional laboratory learning is fairly passive, as the students need to perform the pre-assigned experiments by following instructions provided from the teachers, depriving them of opportunities for self-directed learning, i.e. it cannot cater students with diverse background.

Besides traditional laboratory sessions, some educators start to explore on new routes of laboratory teaching, such as virtual laboratory [2-6]. In these studies, virtual laboratory solved some issues associated with traditional laboratory. These studies showed that virtual laboratory can support science teaching in some ways [6,7]. However, for computer simulations or virtual laboratory, subtle details are easily neglected because of their irrelevance to the main scientific phenomena under consideration. For example, increasing the separation between the sound source and the receiver naturally leads to a reduction in the detected sound intensity (inverse-square law), irrespective to the presence of interference or diffraction effects. The absence of such features convey a naive message that real-life experiments have simple correspondences between the experimental parameters and the observables discussed, and are free from other potential influences. Including such complexities in simulation, while possible, is cumbersome and it involves a tricky balance between highlighting the specific phenomena and the complexity of the real-world situations. Additionally, as simulations always yield 'perfect results', students are deprived of the opportunities to understand how randomness, imperfections and errors can arise in real experiments. For example, randomness in radioactive decay processes is inevitable and can be systematically analyzed, while systematic errors or instrument 
noises can be suppressed with proper experimental techniques. The discussion of such 'imperfections' are also of significance for scientific studies.

Recently, apart from virtual laboratory, remote laboratory experiments, which allow students to access automated setups via the internet, have been suggested to replace the traditional laboratory $[8,9]$. In these remote laboratories, the setups involve simple user interfaces for displaying experimental control and results. Due to the explosive development of internet technology, recently-reported remote laboratories are mostly incorporated with real-time video streaming of the setup. Additional features, such as multi-user control and discussion forum, facilitates the interactions between instructors and students [10]. Using the remote experiments, students can monitor and actively control the setups by themselves through sensors, with real-time video monitoring to visualize the experiment 'in action' [11].

There are also practical concerns that motivated us to look for alternative experiment experiences for students. From September 2009, the educational system in Hong Kong has shifted to a new 3-3-4 system, which consists of threeyears of junior and three years of senior secondary education (graduate with Diploma of Secondary Education DSE), and four years of university education. This is in contrast with the old system of seven-year secondary education plus three years of university education. The first cohort of students educated under the new 3-3-4 system was admitted to our university (PolyU) in 2012. We realized that the students who completed DSE and enrolled into PolyU's physics and engineering programmes have very diverse science background. In a typical 2-hour laboratory session when students are asked to perform controlled experiments, some students finish the laboratory without any difficulty in the allotted time, while others show great difficulty in setting up and finishing the experiment within the assigned time.

Out of the considerations above, we developed a remote laboratory (RemoteLab) platform which can cater for different student backgrounds and provide opportunities for self-directed learning of fundamental principles of physics, before they progress towards more advanced subjects in their course of study. In particular, here we report the development of the optical interference experiment. In the design of our RemoteLab, an internet-based platform is available for students to perform some particular experiments. No specific software is required to run the platform. The flexible learning schedule helps students to increase their learning experience and enhance their understanding of experiments, so students are able to repeat experiments anytime. This allows slow-learning students to repeat the experiments to achieve better results, and perform their experiments anytime, anywhere and as often as they need.

\section{HARDWARE}

We have developed a centralized repository of selected experiments for the platform to improve physics learning experience for our first year undergraduate students. Through remote access via the internet, students operate such setups in a way similar to running the experiment in school laboratories. With the platform, various teaching and learning (T\&L) modes can be adopted as deemed suitable by teachers. For example, teachers can use a particular setup for in-class demonstrations and illustrate important concepts, or assign the experiment as assignments for students' exploration after class.

Our RemoteLab plaform primarily now hosts six experiments (interference of light, Earth's magnetic field, radioactivity, electron charge/mass ratio measurement, ultrasound imaging and photoelectric effect). The architecture of RemoteLab is similar to many of other existing remote laboratory platforms and is illustrated in Fig. 1. Users approach the platform via the webpage of RemoteLab, and access to the web server of RemoteLab platform requires pre-assigned usernames and passwords. The web server allows user access to the interfacing control program in local consoles, which are responsible for manipulation of various components of the experiment setups, data acquisition from sensors and the signal transmission to the remote users. 


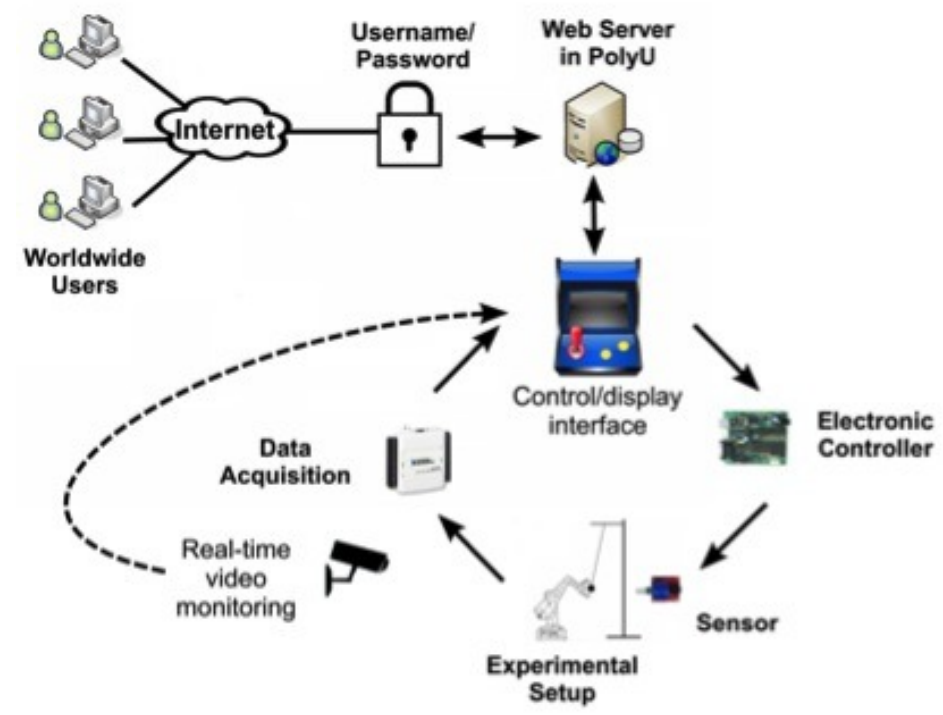

Fig. 1 Schematic diagram showing the concept of real-time, remote-access laboratory experiment platform.

To demonstrate the functionalities of various components in the RemoteLab platform, we showcase here the optical interference setup for illustrating the significance of such incorporations in students' experiment learning experience. The purpose of the experiment of optical interference is to examine the interference patterns formed by laser light after passing through slits of different parameters. Also, the effect of the wavelength of light on the interference pattern can also be examined. Successful generation of the interference pattern require precise positioning of the laser beam on the slits. Although this optical alignment process can be easily achieved by pre-defined movement of the laser, it is a good learning opportunity for students to monitor the processing while doing the experiment using a remote monitoring system.

To allow the students to perform the experiments as if they perform it in the laboratory, the hardware include the following components:

1. Local computer consoles for the control and data acquisition of each experiment setup. Once verified by the log-in system, the remote user, who can be in a school or at home or anywhere else, is directed to the local console of the reserved experiment setup. The console activates the control, viewing and data-logging interfaces for the setup. A user-console communication interface provides the linkage between the remote user and the components. This interfacing programme was built on LabVIEW. To avoid the potential issue of installing drivers at users' computers for operating LabVIEW instruments, LabVIEW Web Service was used for exchange data with web clients (browsers) by sending HTTP requests to specific URLs. Data can be transferred between users (control commands) and remote experiments (experimental results) in $\mathrm{html}$, in a way similar to that exported by the LabVIEW. The time delay during the process is less than 0.5 seconds and should not be noticeable for most users. Most importantly, the html code can be read by all the browsers on various operating systems, without the need of installing specific software. Real-time visualization of the setup (by video streaming, for example) allows monitoring and manipulation of the setup through the interface. This real-time manipulation of the setup provides a path for the students to learn some basic laboratory skills. Finally, data collected are logged and stored in the local console, which can be downloaded to the remote user's computer at the end of the experiment. 


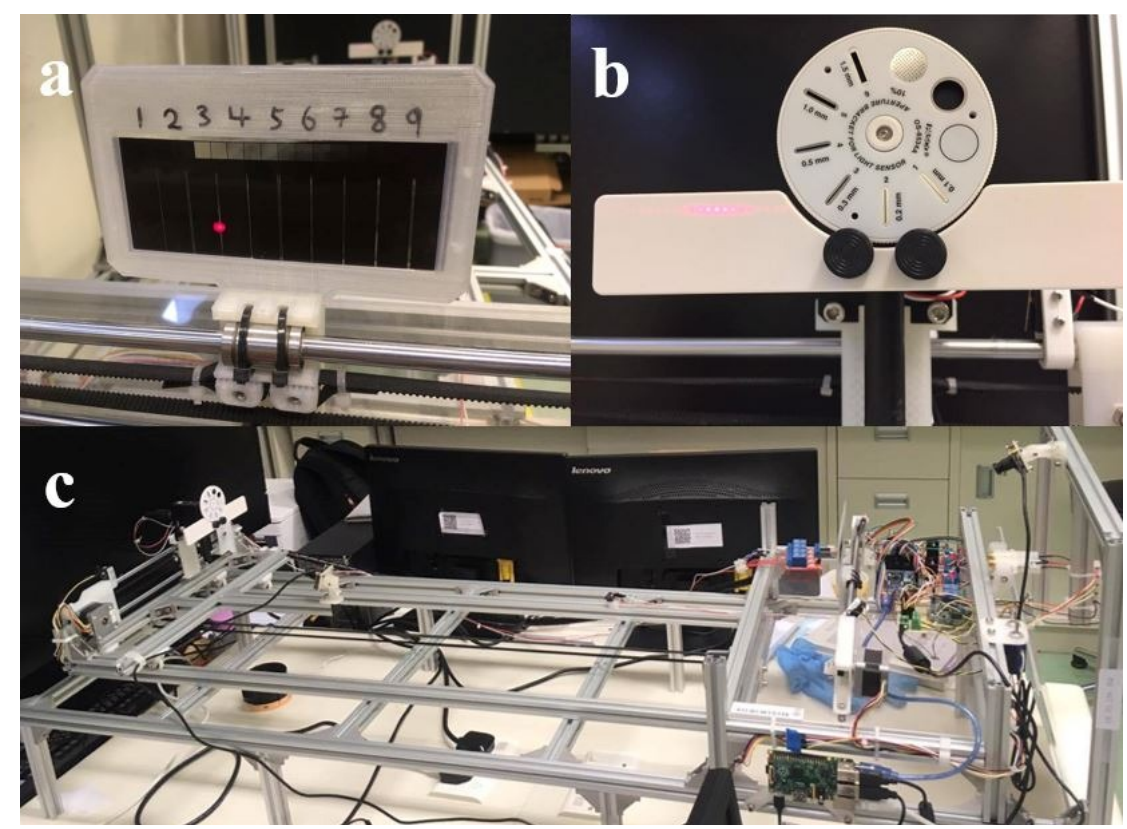

Fig. 2 Components of light interference experiment. (a) Movable slit sets, (b) light intensity sensor with white screen for easy visualization of interference pattern, and (c) complete setup. Notice the web camera at the top right corner of picture for viewing the slits during experiment.

2. Automation is necessary for adjusting various experimental parameters. Consider the case of the light interference experiment. The main components of the experiment include light sources (two laser diodes, one in green and one in red), light sensors, and slits of different widths and separations. Mechanical and electronic devices are required to sweep the light sensor across the interference pattern generated for collecting data, as well as switching between different slit pairs. In the setup as shown in Fig. 2, the motion for various degrees of freedom (translation of the slits in Fig. 2(a) and light sensor in Fig. 2(b)) are provided by corresponding stepper motors, which are in turn triggered by the console via a controller box. Besides, signals detected by the light sensor need to be collected by the console via data acquisition cards for further processing.

3. A set of viewing ports for real-time visualization of key experimental parameters. Particular attention was paid on the parts concerning the experimental input parameters and the final results. Considering again the case of the prototype setup in Fig. 2(c), video cameras are installed to monitor the slit position (right hand side of the figure) and the interference pattern generated on the white plastic screen/light sensor (left hand side).

\section{ACCESSORIES}

While the hardware is crucial for the RemoteLab, some basic but significant functions are also available in the RemoteLab platform [12], as listed in the following.

1. A log-in system which allows registered users to gain access from a remote computer to the experiment platform in PolyU and perform the experiments. The log-in system permits users to make reservations of time slots for conducting specific remote experiments, and provide necessary security features to ensure access to the system only by registered members.

2. A booking system that allows students book the experiments at the own time i.e. this platform takes away the constraints of time, location (Fig. 3(a)). As we have mentioned that the students have diverse background and learning rates, this platform can create opportunities for multiple attempts to help students master the concepts through experimentation. 
3. A webpage which interfaces the user and the local console (Fig. 3(b)). All user commands for controlling the console (log in system, experiment setup, data acquisition and retrieval) and displaying information (video streaming, experimental results) are accessible via the webpage.

4. Short videos on the procedures of operating the setups (Fig. 3(c)). 'Sample videos' were prepared, illustrating the physical phenomena expected to be observed from the experiments.

5. Background information on the physical phenomena related to particular experiments were provided in dedicated webpages, accessible directly from the experiment site. Separate materials discussing the technical details and suggested teaching strategies were available for the teachers on separate webpage.

6. Laboratory worksheets were prepared to guide the students through the experiments with structured questions. Through these worksheets, students practice their skills in making observations, doing analysis and drawing conclusion by performing the tasks sequentially. With the setups and provided brief guidelines, students can design their own investigations by making refinement on the setups. Indeed, such instruction sheets are to kick-start the brainstorming process among students in deciding their project objectives and experimental methods.
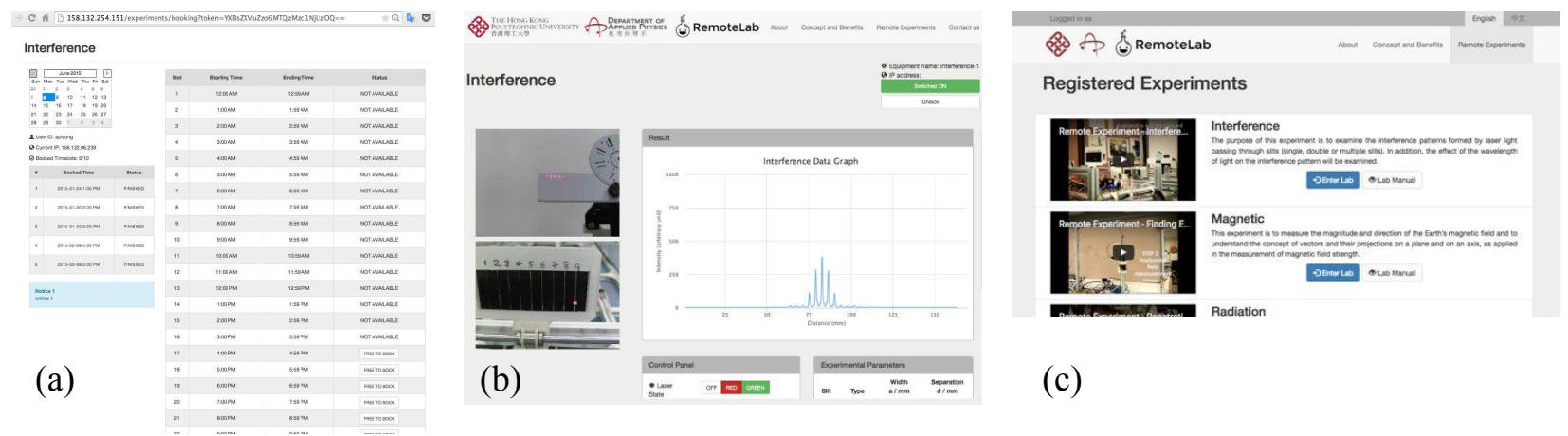

Fig. 3 Important features on RemoteLab platform. (a) Booking system for reservation of timeslots. (b) Main experiment page for controlling and monitoring the setup, as well as display and handling of collected data. (c) Collection of introductory videos for various experiment setups.

\section{EVALUATION}

To gauge the educational impact of the RemoteLab platform on students' learning of interference effect, the interference experiment was prescribed to two groups of students, namely a group of form five students from a local secondary school, and a group of freshman undergraduates in the Department of Applied Physics of PolyU.

\section{PolyU freshman students' survey}

The survey was conducted in 2016 to collect users' opinion on RemoteLab's interference experiment, including the experimental setup, booking system, user interface and other related aspects. 109 students completed a 15-question survey (Table 1) that used a 5-point Likert scale from 1 (Strongly Disagree) to 5 (Strongly Agree). We note that the survey was different from the secondary school students' survey (Table 2, to be discussed in the next paragraph) as the survey for undergraduates were primarily for optimizing the setup. 


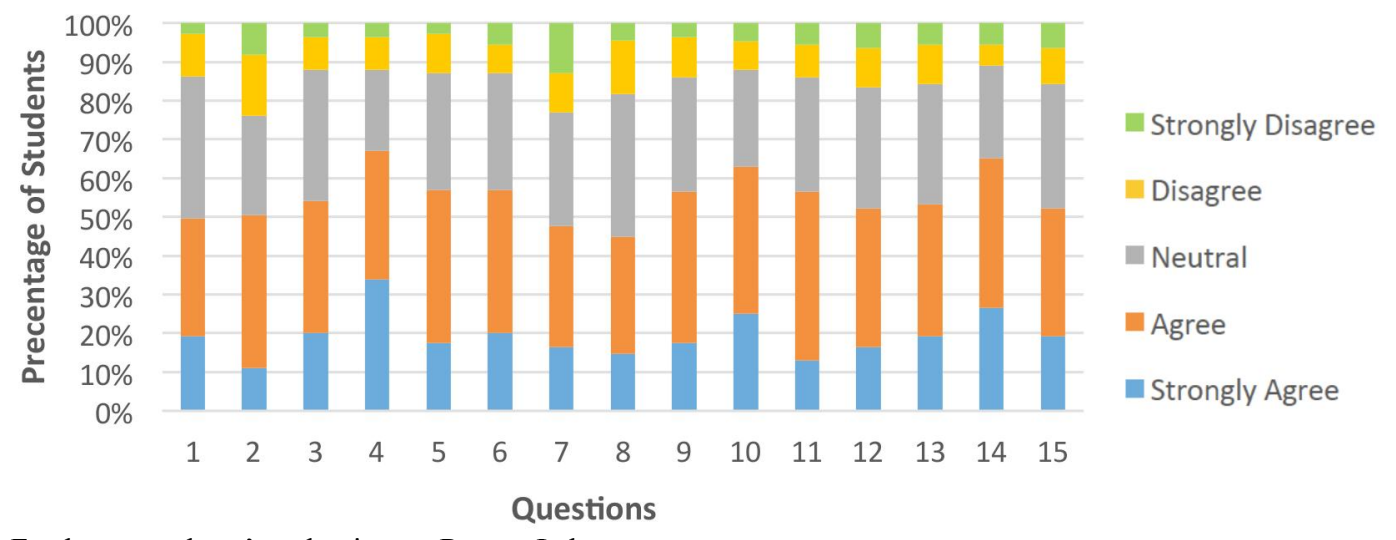

Fig. 4 Freshman students' evaluation on RemoteLab.

Table 1. Survey used for evaluating on RemoteLab by undergraduates in AP, PolyU.

Experimental Setup
Q1. Interference of Light is suitable for remote control experiments
Q2. Experiment setup can be visualized clearly from screen
Q3. More viewpoints are necessary
Booking System
Q4. Easy to use the booking system
Q5. Sufficient guidance provided to book RemoteLab
Q6. Time allocated for each session is appropriate
Q7. Prefer to use email to notify user of booking time
User Interface
Q8. Online instructions to conduct experiments are clear
Q9. Webpage design is user-friendly
Q10. Easy to enter the Remote Lab for experiment
Q11. Instructions to use the Remote Lab are clearly stated in the lab manual
Overall Comment
Q12. It is an effective way to conduct a laboratory session
Q13. The experiment can be completed smoothly
Q14. It's a good learning experience
Q15. RemoteLab serves as a supplementary to a normal Laboratory session

As shown in Fig. 4, more than $80 \%$ of students chose "Neutral" or 'Agree/Strongly Agree' on questions 4 and 8. The result of question 4 (mean $M=3.85$, standard deviation $\sigma=1.096$ ) suggests that it is easy to use the booking system of RemoteLab, and question $8(M=3.37, \sigma=1.042)$ shows that the online guidance to conduct experiment are clear. Also, almost $84 \%$ of participants chose "Neutral" or above on question 12, which means they likely agreed RemoteLab is an effective way to conduct a laboratory session. Close to $70 \%$ of participants chose "Agree" or "Strongly Agree" on question 14, and it indicates that 71 out of 109 students agreed RemoteLab is a good learning experience.

\section{Secondary school students' survey}

For the survey on secondary school students, we attempted to measure students' understanding on the specific physics topics by questionnaire. The questionnaire asks for some basic experiences such as students' reactions about user interface, support materials, experiment and platform setup. To thoroughly understand the benefits and improvement of RemoteLab, the questionnaire also focuses on students' learning process about doing the interference experiment, and the evaluation indicates students' expectation and suggestion about their platform and experiment setup through their knowledge. 
Table 2. Survey used for evaluating on RemoteLab by secondary school students. Unless specified, the questions are rated on a 5pont Likert scale (1: Strongly Disagree; 5: Strongly Agree).

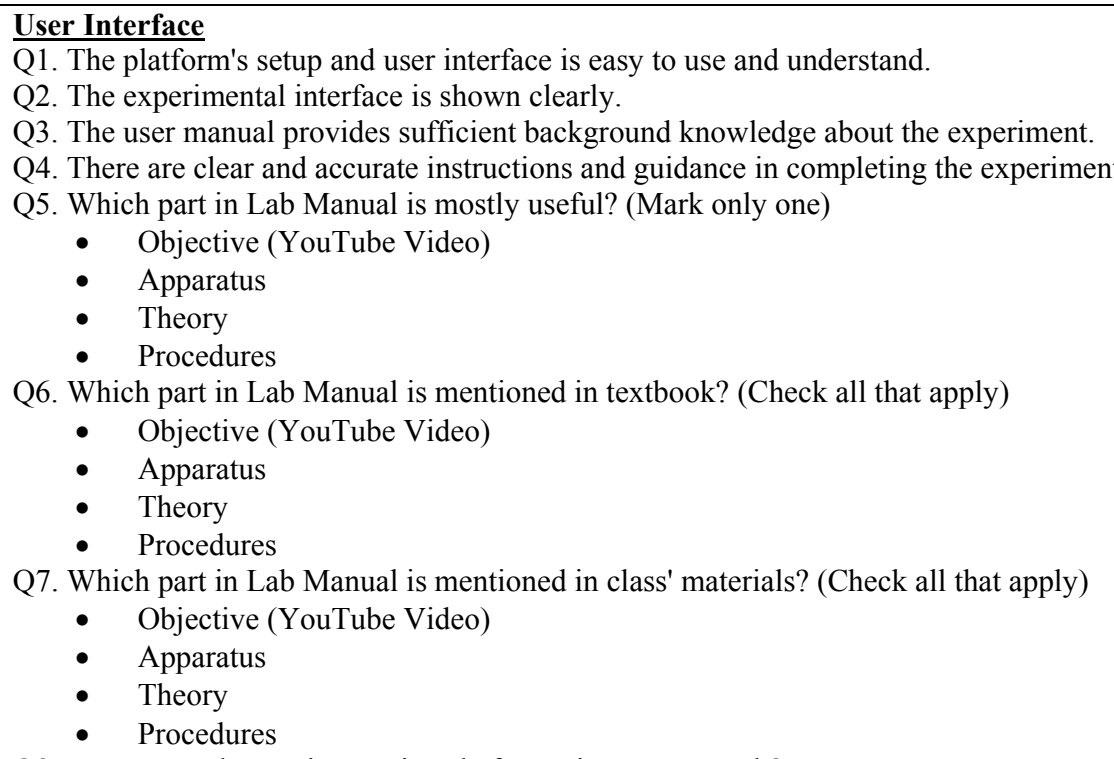

Q8. Do you need more instructions before using RemoteLab?

Booking System \& Experiment Setup

Q9. It is easy to book and enter lab session.

Q10. There is sufficient time to complete all the tasks within the reserved time slot.

Q11. The experiment functions smoothly.

Q12. The viewing ports allow you to visualize the setup clearly.

Q13. It is easy to download the data and result for analysis.

Q14. The experimental results obtained in a "good quality" way.

Q15. The data you obtained is able to fit in the scientific theory or phenomenon.

\section{Learning Process}

Q16. What was your expectations before using RemoteLab? (Check all that apply)

- Hands-on experimental experiences

- $\quad$ Problem-solving skills

- Analysis

- Enhance theoretical and conceptual knowledge

- $\quad$ Thinking skills

Q17. Did the platform meet your expectations?

Q18. How did the result and data of experiment satisfy your expectations? (1-Very Dissatisfied - 5-Very Satisfied)

Q19. How did the supporting materials (lab manual) satisfy your expectations? (1-Very Dissatisfied - 5-Very Satisfied)

Q20. How did the process of performing experiment satisfy your expectations? (1-Very Dissatisfied - 5-Very Satisfied)

Q21. How did the experiment setup and user interface satisfy your expectations? (1-Very Dissatisfied - 5-Very Satisfied)

Q22. Did you gain better understanding about the scientific theory after doing experiment?

Q23. In this experiment, I observed these following details: (Check all that apply)

- $\quad$ Effects of wavelength (color) on interference patterns

- Effects of slit separation on interference pattern

- Relationship between fringe separations

- The different interference patterns of single, double and multiple slits

\section{Comments}

Q24. The RemoteLab is a good learning experience.

Q25. The RemoteLab provides an effective and advanced learning method to conduct a laboratory session.

Q26. The RemoteLab increases the opportunities of performing experiment.

Q27. The RemoteLab enhances your learning motivation. 
Q28. How would you rate your experience regarding this activity? (1-Very Poor - 5- Excellent)

Q29. Would you recommend this platform to others?

Q30. Any suggestions and recommendations

Secondary school students' survey was conducted in 2017. 11 participants completed the survey with 30 items, including various types of questions such as closed format, rating scale, likert scale (from 1-Disagree to 5-Agree), and dichotomous questions. In these 30 items of the survey, we focused on 4 different topics, namely user interface, booking system, experiment setup, and learning process. Students' experiences in RemoteLab and how this platform influenced their learning processes was evaluated.

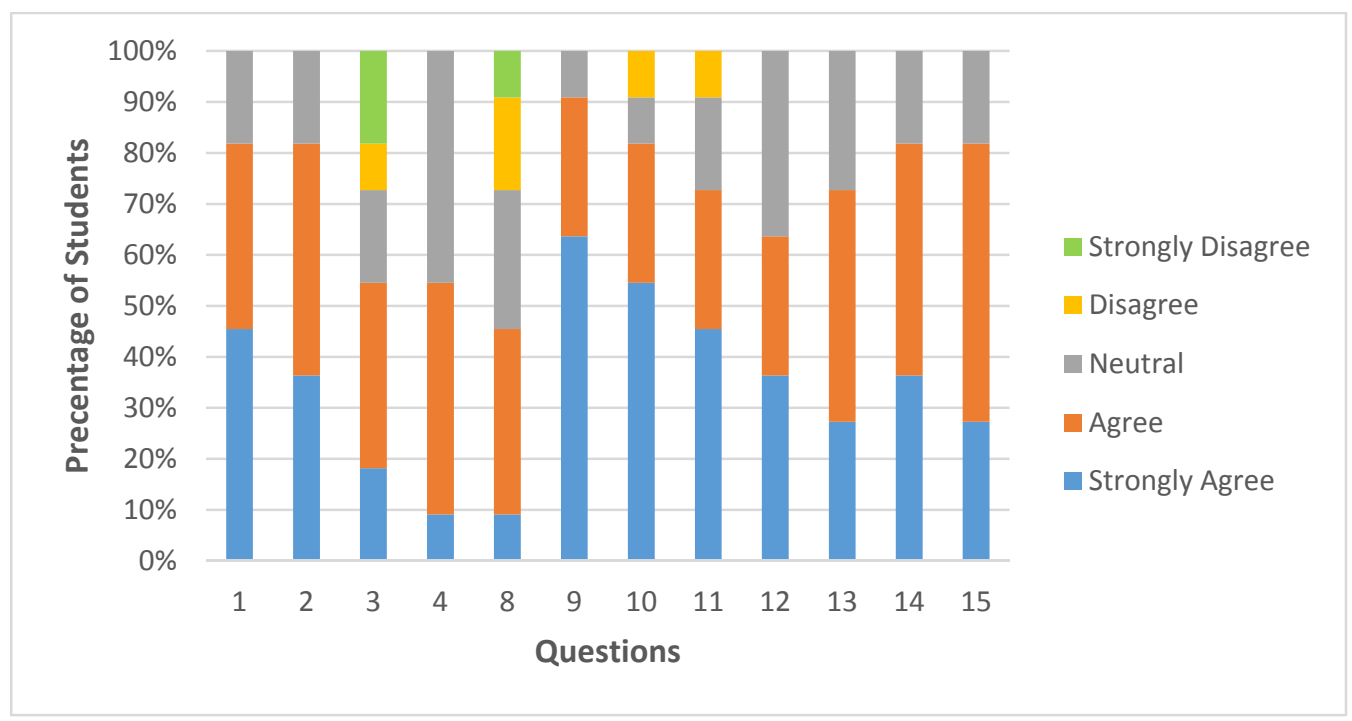

Fig. 5 Secondary school students' evaluation on RemoteLab.

As shown in Fig. 5, more than $80 \%$ of participants chose "Agree" and "Strongly Agree" in question $1(M=4.27, \sigma=$ 0.79), which means most of them likely agreed the setup of RemoteLab platform and user interface is easy to use and understand. In addition, question 9 shows that $90 \%$ of participants $(M=4.55, \sigma=0.69)$ are mostly agreed it is easy to book and enter lab session. On question $12(M=4, \sigma=0.89)$ and $13(M=4, \sigma=0.77)$, all of the 11 students chose neutral or above, and none of them chose "Disagree" or "Strongly Disagree." It shows all of the participants believed that the viewing ports of experiment setup showed clear images of the setup, and the data was easy to download for analysis. For the experiment setup (question 12-15), 100\% of participants chose "Neutral" or above; none of them chose "Disagree" or "Strongly Disagree". In particular, more than 80\% of participants chose "Agree" and "Strongly Agree" for question 15, which means they mostly agreed the data could fit the scientific theory or phenomenon. 


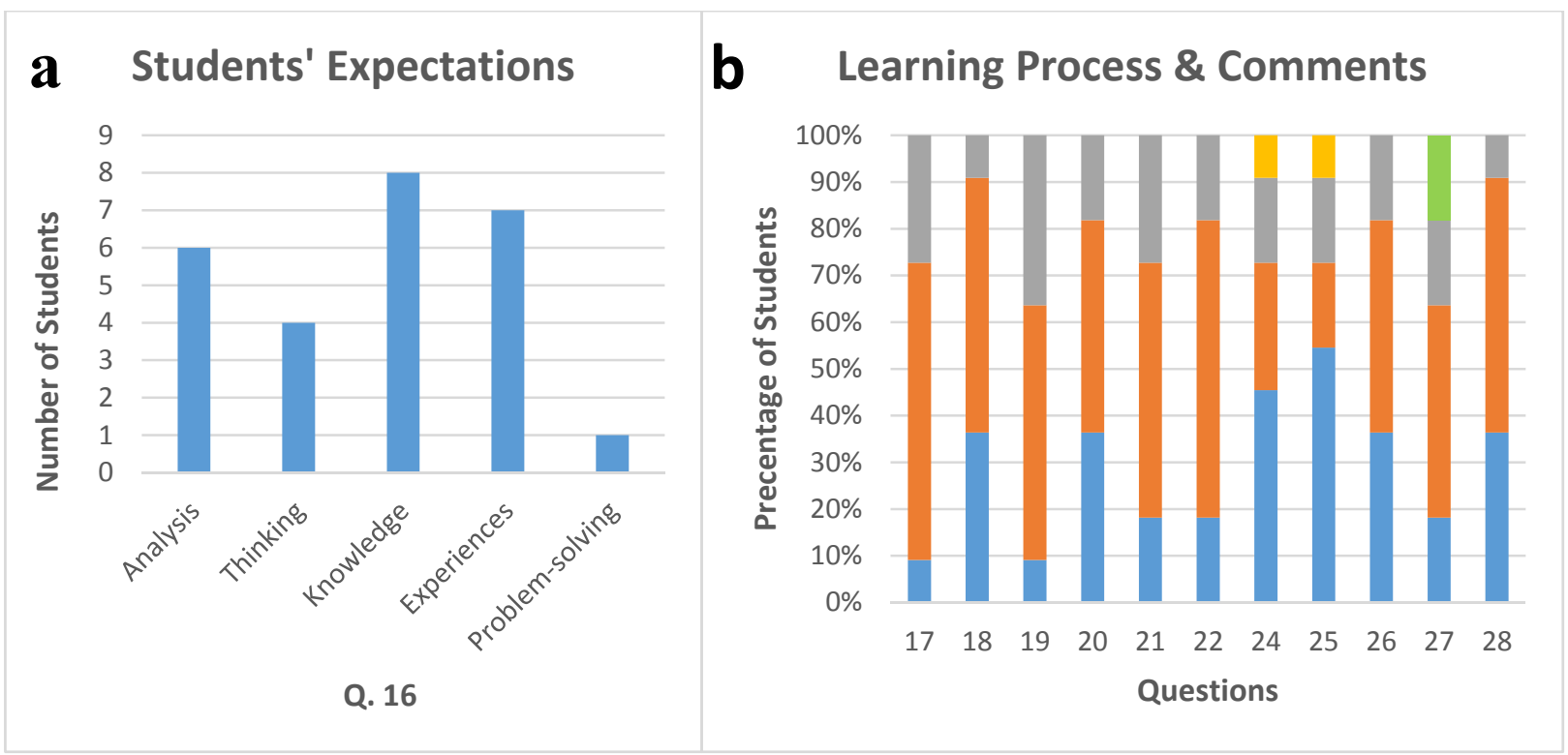

Fig. 6. Secondary school students' evaluation on RemoteLab (Learning Process \& Comments), based on their responses on question 16 (a) and questions $17-28$ of the survey in Table 2.

Fig. 6a and 6b show the analyses of all items used for evaluating students' learning process and their comments on using RemoteLab. Question 16 of the survey aimed at probing the students' expectations before using RemoteLab. Based on the graph, 8 out of 11 participants chose "Knowledge". Questions 17 to 21 were used to show students' expectations in different aspects of RemoteLab. 60\% - 90\% of participants chose "Agree" and "Strongly Agree" on these items.

Concerning the last two (open-ended) questions in the survey to secondary school students, most of the students agreed that Remote Lab has certain advantages. They appreciated the flexibility of Remote Lab. They were able to operate setups anytime and anywhere through the internet. The booking system was simple and user friendly. Users were able to book more than one lab session, allowing them to conduct the experiments more than once and obtain better results compare with traditional lab. Moreover, due to the flexible learning schedule, participants could conduct experiment and discuss with groupmates outside regular and specific time of traditional lab sessions. They also commented that the instructions were easy to follow and demonstration videos were clear, which were useful for students with weak scientific background.

However, half of the students experienced difficulty in booking lab sessions. Lab session for some experiments were fully occupied near the deadline and one participant mentioned he could not login even when he booked the lab session. Peer support was not strong on the RemoteLab platform, as they cannot discuss with other team members. Sometimes, the motivation to conduct experiments was low as the setup was distant and tedious on clicking buttons repeatedly. Two of the interviewed students showed ignorance of theories and meaning of procedures. They agreed that it would be better if there were lectures or relevant materials covering the knowledge of RemoteLab. Some students suggested they might acquire more hands-on experience if they could 'build' the experiment setups by themselves.

There are some discussions in Fig. 4, 5 and 6. For instance, as shown in Fig. 4, close to $24 \%$ of freshman students thought experiment setup cannot be visualized clearly from screen. According to students' feedback, RemoteLab could be improved by setting more viewports that students could visualize the experiment setup much easier. As shown in Fig. 5, question 3 indicates that there are almost 30\% of participants think the lab manual did not provide sufficient background knowledge about experiment. Students suggested the provisions of more instructions and guidance, which enhances their understanding of experiment theoretically and practically and facilitates them to 
conduct the experiment. On question 10 and $11,10 \%$ of participants disagreed there is not sufficient time to complete all the tasks within the reserved time slot and disagreed the experiment functions smoothly. As shown in Fig. $6 \mathrm{~b}, 2$ out of 11 participants $(M=3.45, \sigma=1.37)$ might think RemoteLab does not help them to enhance their learning motivation.

In order to improve the Remote Lab, participants suggested to restrict the number of sessions that can be reserved by each student per day. They proposed to modify the operation procedures of RemoteLab, increase the complexity and authentic feeling of operation instead of simply clicking the buttons. They prefer setting up the apparatus by themselves to enhance their attention in operation and hence developing a stronger impression on the lab experience. They agreed that changing the layout of website with icons looking like real equipment (such as 'turning knob' button to 'switch on/off') equipment would enhance students' interests and understanding on the experiment procedures.

\section{CONCLUSIONS}

In summary, we have developed a remote experiment platform which enables students to conduct experiments everywhere at all times. The particular experiment of interference of light was showcased in this paper, with the details of the hardware and accessories introduced. The experiment was piloted in two groups of students, and the users were generally positive towards the idea of remote experiments. It is anticipated that the platform can be further elaborated for other disciplines of science education, and it's potentials awaits to be systematically explored.

\section{ACKNOWLEDGEMENTS}

The work was supported by the Hong Kong Polytechnic University (LTG12-15/422B, eLF13-16/8CJ3) and the Quality Education Fund, HKSAR (2013/0127). 


\section{REFERENCES}

1. Sunal, D.W., Wright, E.L. and Sundberg, C., [The impact of the laboratory and technolody on learning and teaching science K-16], Information Age Publishing, Charlotte, North Carolina, 1-27 (2008).

2. Wiesner, T.F. and Lan, W., "Comparison of Student Learning in Physical and Simulated Unit Operations Experiments," Journal of Engineering Education. 93(3): 195-204 (2004).

3. Finkelstein, N.D., et al., "When learning about the real world is better done virtually: A study of substituting computer simulations for laboratory equipment," Physics Education Research. 1(1): 010103 (2005).

4. Olympiou, G. and Zacharia, Z.C., "Blending physical and virtual manipulatives: An effort to improve students' conceptual understanding through science laboratory experimentation," Science Education. 96(1): 21-47 (2012).

5. $\quad$ Horton, J.J., Rand, D.G. and Zeckhauser, R.J., "The online laboratory: conducting experiments in a real labor market," Experimental Economics. 14(3): 399-425 (2011).

6. Poindexter, S.E. and Heck, B.S., "Using the web in your courses: what can you do? what should you do?" IEEE Control Systems. (1999).

7. Trundle, K.C. and Bell, R.L., "The use of a computer simulation to promote conceptual change: A quasiexperimental study," Comput. Educ. 54(4): 1078-1088 (2010).

8. Diwakar, A., Poojary, S. and Noronha, S.B., "Virtual labs in engineering education: Implementation using free and open source resources, " IEEE. (2012).

9. Tudevdagva, U., Ayush, Y., and Baatar, B., "The Virtual Laboratories Case Study in Traditional Teaching and E-Learning for Engineering Sciences," UMEDIA. (2014).

10. Lowe, D., Newcombe, P. and Stumpers, B., "Evaluation of the Use of Remote Laboratories for Secondary School Science Education," Research in Science Education. 43(3): 1197-1219 (2013).

11. Shelke, S., et al. "A Remote lab for real-time digital signal processing," (EDERC). (2012).

12. https://remotelab.ap.polyu.edu.hk/ 